\title{
THE FAITHFULNESS QUESTION FOR THE BURAU REPRESENTATION
}

\author{
JOHN MOODY
}

(Communicated by Ronald J. Stern)

\begin{abstract}
We consider the Burau and Gassner representations of the braid groups $B_{n}$. A sufficient condition for faithfulness, involving just a pair of arcs, is shown to be necessary as well for all but at most two values of $n$. In the Burau case, this implies nonfaithfulness for $n \geq 10$.
\end{abstract}

Alexander showed in [6] that any knot $K$ can be made by connecting the ends of some braid $b$. The Alexander polynomial $\Delta(K)$ was defined in terms of a certain matrix $A$ of relations. In 1926, Alexander found that $\Delta(K)$ does not uniquely determine the knot $K$ [1].

Subsequently, Burau wrote down, for each braid $b$, a matrix of Alexander relations of the form $A(b)-1$ for the associated knot or link, such that the matrix of a composite $b b^{\prime}$ satisfies

$$
A\left(b b^{\prime}\right)=A(b) A\left(b^{\prime}\right) .
$$

For generic values of the variable, Burau's representation (modulo a line) is Zariski dense, so its exterior powers are distinct irreducible representations. Up to a constant, the Alexander polynomial is the alternating sum of their characters on the positive braids, and, up to normalization, it agrees with the unique extension to a virtual character on the braid group $B_{n}$.

The question of faithfulness of Burau's representation, equivalent to that for $\Delta$ viewed as a virtual character, was then intensively studied first systematically by Magnus and his students (see, for instance, Magnus' collected works [2]). Some early attempts are now subsumed in Long's result that the faithfulness of any representation can be detected on the center and any noncentral normal subgroup of the pure braid group [3].

On the other hand, the sets of braids with given closure were found to be equivalence classes under certain so-called Markov moves [4]. These issues converge in Birman's monograph [5], which states that conceivably the Burau representation could become unfaithful for $n \geq 5$, and gives a rigorous proof of the sufficiency of the Markov moves.

Received by the editors March 5, 1992.

1991 Mathematics Subject Classification. Primary 57M25; Secondary 20F36. 
Here we will give a necessary and sufficient condition ${ }^{1}$ for the faithfulness of the Burau representation and a related representation called the Gassner representation. This paper was circulateci in preprint form and delivered at the Queen Mary College topology discussion group, and a talk in Bristol, except the conditions were both included as open questions. We now include simple counterexamples to the Burau condition, which I noticed in the week following the second talk. Long and Paton have each, independently, found counterexamples to the condition for smaller values of $n$.

Our best example is a somewhat complicated braid in $B_{10}$, which I reduced to nine strands. This example was analyzed on the computer by Morton and Strickland, using the algorithm of Morton and Short to calculate the 2-variable polynomial. This verified that the braid was a Burau counterexample, gave the first proof that the associated link is also not trivial, and showed that there are braids in the kernel of the Burau representation which are not in the kernel of Jones' representation. Conversely, results of Long show that for some components of the Hecke algebra representation, the kernels contain the Burau kernel [7].

In the work reported here, computers were not used. Between moving to the United Kingdom in 1988, and writing down the 9-strand example in March, I have not had any occasion to use or write any computer programs. It is reported by some authors that the Gassner representation should be faithful for all $n$. I will at least conjecture that no counterexample has fewer than a thousand crossings, based on drawings by myself and Mark Paton, but nothing in our work suggests that the situation could not change for much larger knots.

\section{INTRODUCTION}

In $\mathbb{C} \times(-\infty, 0]=\{(z, r)\}$, consider the vertical lines $z=1,2, \ldots, n$. We shall think of a braid as the result of applying any bounded, level-preserving isotopy $\Phi_{t}$ to the embeddings of the lines which brings the endpoints in $\mathbb{C} \times 0$ back to $1,2, \ldots, n$ in some possibly permuted order. Under this correspondence, the so-called pure braids are the ones in which the associated permutation of the endpoints is trivial. Two braids are thought to be equivalent if they differ only by a bounded, level-preserving isotopy fixing the endpoints.

At level zero, the isotopy which creates a braid amounts to a motion of the $n$ distinct endpoints in $\mathbb{C}=\mathbb{C} \times 0$. It is well known both that the braid can be recovered up to equivalence from this motion, and that the motion extends to a compactly supported isotopy of the identity diffeomorphism of $\mathbb{C}$.

The composite of braids, once one has modified the second to be nontrivial

\footnotetext{
${ }^{1}$ To be precise, the condition is necessary and sufficient for all but at most two unspecified values of $n$ (see the statement of the Theorem). The higher commutators in the proof fill a gap in my first draft, which was found by J. Birman. In an upcoming conference proceeding, J. Birman, D. Long, and myself show that the analogous faithfulness condition for homology of a general braid-equivariant local system works for all but at most $2(r-1)$ values of $n$, where $(r-1)$ is the faithfulness range of the braid action on the fiber of a fixed point. This gives a faithfulness condition for the irreducible summands of the Lawrence representations, but not for all $n$.

In the Burau and Gassner cases (when $r=2$ ), the higher commutators are superseded by a geometric result of D. Long; by an ingenious argument, the D. Long and M. Paton paper actually obtains necessary and sufficient conditions for all $n$. It is hoped that the Long-Paton method may somehow supersede higher commutators for larger $r$, but this is not known. A unified treatment of these further issues in a research paper is not currently available.
} 
only when $r<r_{1}, r_{1}$ being chosen so that the first is nontrivial only for $r>r_{1}$, consists of the points of the first braid which are above level $r_{1}$, together with the points of the second that are below.

It is helpful to imagine what you would see from an eye located at $\left(z_{0}, r_{0}\right)$ with $\operatorname{Im}\left(z_{0}\right)<0$ and $r_{0}>0$. Projecting the braid $\mathbb{C} \times 0$ through all lines through this point produces a photograph of the braid consisting of curves $p_{1}, \ldots, p_{n}$ emanating in order of angle from $z_{0}$ in the lower half-plane, and ending at $1,2, \ldots, n$ in some order. Naturally, we should choose the point $\left(z_{0}, r_{0}\right)$ to minimize the number of crossings we see. In fact, allowing an additional compactly supported, level-preserving isotopy fixing the endpoints, we can remove all crossings. Let the original motions of the endpoints extend to an isotopy $\psi_{t}(z)$ of the identity on $\mathbb{C}$, which fixes all points as far away as $z_{0}$. Writing $t=1 /(1-r)$ and $u=\left(r_{0}-r\right) / r_{0}$, the braid whose points are $\left(z_{0}+u\left(\psi_{1}\left(t j+(1-t) z_{0}\right)-z_{0}\right), r\right)$ will appear to have no crossings, as it will project to the union of the curves

$$
p_{j}(r)=\psi_{1}\left(t j+(1-t) z_{0}\right), \quad j=1,2, \ldots, n .
$$

These curves approach $z_{0}$ as $r$ tends to $-\infty$, and take values of $1,2, \ldots, n$ when $r=0$.

In the discussion below, if $p$ is any smooth arc in $\mathbb{C}-\{1, \ldots, n\}$ from $z_{0}$ to one of $1, \ldots, n$, the symbol $\partial(p)$ will denote the smooth simple closed curve based at $z_{0}$ which traces the boundary of a small neighborhood of $p$ in the counterclockwise direction.

Here is Kohno's definition of the Burau matrix [8]. For any smooth simple closed curve $\gamma$ based at $z_{0}$ in $\mathbb{C}-\{1, \ldots, n\}$, let

$$
w(\gamma)=\int_{\gamma}(z-1)^{\lambda_{1}} \cdots(z-n)^{\lambda_{n}} d z .
$$

Although there are infinitely many choices of $\gamma$, and the functions $w(\gamma)$ even span an infinite-dimensional complex vector space $W$, this vector space is free of finite rank $n$ when viewed as a module over the ring $R$ of finite Fourier series in the variables $\lambda_{1}, \ldots, \lambda_{n}$.

Letting $E_{i}$ denote the straight path from $z_{0}$ to $i$, one observes that the functions $e_{i}=w\left(\partial\left(E_{i}\right)\right)$ form a basis of the module. Let $w_{i}(\gamma)$ be the $i$ th coefficient of $w(\gamma)$ in the basis. The action of a braid $b$ whose semi-infinite strands appear as the finite arcs $p_{1}, \ldots, p_{n}$ in $\mathbb{C}-\{1, \ldots, n\}$ is given on the basis elements by the formula

$$
b e_{j}=w_{1}\left(\partial\left(p_{1}\right)\right) e_{1}+\cdots+w_{n}\left(\partial\left(p_{n}\right)\right) e_{n} .
$$

This extends to an action on all of $W$ by the following rule of semilinearity: the action of $b$ is $\mathbb{C}$-linear, and if $w \in W$ and $r \in R$, then

$$
b r w={ }^{b} r b w
$$

where ${ }^{b} r$ is obtained by replacing each occurrence of $\lambda_{j}$ in $r$ by $\lambda_{p_{j}(0)}$.

Up to isomorphism, these rules describe the so-called Gassner representation at any system $T_{1}, \ldots, T_{n}$ of complex numbers whose permutations are all induced by automorphisms of $\mathbb{C}$, once we choose $\lambda_{j}$ such that $T_{j}=e^{2 \pi i \lambda_{j}}$. This representation is semilinear for the action of the braid group by a suitable group 
of Galois automorphisms. As a special case, we obtain the Burau representation up to isomorphism when all $T_{i}$ equal a fixed complex number $T$.

One can read-off the $w_{j}(\gamma)$ by the type of calculation formalized by Fox. Let $f$ be the characteristic function of the upper half-plane $\mathbb{H} \subset \mathbb{C}$. Let $\log _{0}$ be the principal branch of $\log$, a single-valued function with a discontinuity along the positive real axis, satisfying $\log (i \pi)=-1$. Let $h(z)$ be the multiple-valued function on $\mathbb{C}-\{1, \ldots, n\}$ given by $h(z)=\lambda_{1}\left(\log (z-1)-\log _{0}(z-1)\right)+\cdots+$ $\lambda_{n}\left(\log (z-n)-\log _{0}(z-n)\right)$, and for $j=1, \ldots, n$ let $\omega_{j}$ be the differential form

$$
\omega_{j}=(\dot{\mathrm{i}} / 2 \pi i) f(z) e^{h(z)}(d \log (z-j)-d \overline{\log (z-j)}),
$$

which becomes well defined on a suitable cover. Reading around a smooth simple closed curve $\gamma$ based at $z_{0}$, the integral of $\omega_{j}$ tabulates $w_{j}(\gamma)$, counting plus or minus some monomial in the $e^{2 \pi i \lambda_{s}}$ each time $\gamma$ crosses the verticle ray $\operatorname{Re}(z)=j, \operatorname{Im}(z)>0$. In fact $w_{j}(\gamma)$ is $e^{-2 \pi i\left(\lambda_{1}+\cdots+\lambda_{j}\right)}$ times this integral.

In particular, $\gamma$ cannot be isotoped off the vertical ray, by an isotopy fixing the basepoint, unless $w_{j}(\gamma)$ is zero. In other words, $w_{j}(\gamma)$ is an obstruction to isotoping $\gamma$ not to intersect the ray $\operatorname{Re}(z)=j, \operatorname{Im}(z)>0$.

Since there is no possibility of confusion, we write $w_{j}(p)=w_{j}(\partial p)$ whenever $p$ is a path from $z_{0}$ to one of $1,2, \ldots, n$. When $j \neq p(0)$, one can use $p$ itself for the path of integration, or the strand projecting to $p$ in some braid of interest, provided one is willing afiterwards to multiply by $\left(1-e^{2 \pi i \lambda_{p(0)}}\right)$.

\section{RESUlt}

From now on let $\lambda_{1}, \ldots, \lambda_{n}$ be complex numbers such that

$$
\left\{\begin{array}{l}
\text { (a) If the } T_{j}=e^{2 \pi i \lambda_{j}} \text { are unequal, any permutation among the } \\
\text { is induced by an automorphism of } \mathbb{C} . \\
\text { (b) The } T_{j}-1 \text { do not generate the linit ideal in the subring of } \\
\mathbb{C} \text { generated by the } T_{j} \text { and } T_{j}^{-1} \text {. }
\end{array}\right.
$$

Condition (a) guarantees that we can calculate the matrix of a product of braids from the matrices of the separate braids, while (b) allows us to forego making separate provision for the case $j=p_{s}(0)$, which seems to have no clear geometric interpretation anyway. For, $w_{j}\left(p_{s}\right)=1$ modulo $T_{1}-1, \ldots, T_{n}-1$ then, so when (b) holds it cannot be zero, consistent with the fact that $p_{s}$ cannot be isotoped away from its own endpoint.

Let us begin again with the situation where we are considering a loop $\gamma$ in $\mathbb{C}-\{1, \ldots, n\}$ based at $z_{0}$. Assume now that, among all the points on our $\gamma, z_{0}$ is a point of minimum possible real-coordinate value. In this situation, we assert that, for all but at most three values of $n$, the obstruction $w_{j}(\gamma)$ is effective if and only if the Gassner representation of the braid group $B_{n}$ is a faithful representation.

To be more precise, and to decrease the gap of three to our knowledge, it helps to now restrict attention to $\gamma$ of the form $\partial p$, where $p$ is a simple path from $z_{0}$ to one of $1,2, \ldots, n$ in $\mathbb{C}-\{1, \ldots, n\}$, and $z_{0}$ is again a point of minimum real-coordinate value on $p$. Let us view $j$ as a variable which ranges over the numbers $1,2, \ldots, n$. 
Theorem. Let $T_{1}, \ldots, T_{n}$ be either variables or complex numbers subject to $(*)$. As usual, set the $\lambda_{s}$ in the definition of the $w_{j}(p)=w_{j}(\partial p)$ such that $e^{2 \pi i \lambda_{s}}=T_{s}$.

(i) If $w_{j}(p)$ is an effective obstruction to isotoping any such $p$ in $\mathbb{C}-$ $\{1, \ldots, n\}$, by an isotopy fixing $z_{0}$, not to intersect the ray $\operatorname{Re}(z)=j, \operatorname{Im}(z)>$ 0 , then the Gassner representation of $B_{n}$ is faithful at $T_{1}, \ldots, T_{n}$.

(ii) If $w_{j}(p)$ is not an effective obstruction to isotopy, then the Gassner representation of $B_{n+2}$ is not faithful at $T_{1}, \ldots, T_{n}$.

Corollary. Let $T_{1}=\cdots=T_{n}=T$ be a variable or a complex number subject to $(*)$. Set the $\lambda_{i}$ in the definition of the $w_{j}(p)$ as usual.

(i) If $w_{j}(p)$ is an effective obstruction to isotoping $p$ by an isotopy fixing $z_{0}$, not to intersect the ray $\operatorname{Re}(z)=j, \operatorname{Im}(z)>0$, then the Burau representation of $B_{n}$ is faithful at $T$.

(ii) If $w_{j}(p)$ is not an effective obstruction to isotopy, then the Burau representation of $B_{n+2}$ is not faithful at $T$.

Proof of the Theorem. If the Gassner matrix is the identity, then $w_{j}\left(\partial p_{k}\right)=0$ for $j \neq k$. Look at the braid from the point $\left(z_{0}, r_{0}\right)$ as in the introduction, in such a way that it appears to have no crossings. Assuming the obstructions are effective, any one arc can be isotoped into a straight arc $z=k$. Moreover, since this extends to an ambient isotopy, we can again assume that there appear to be no crossings. In general, the number of crossings in front of a straight strand $z=$ $k$ in $\mathbb{C} \times(-\infty, 0]$, when viewed from $\left(z_{0}, r\right)$, will remain constant or decrease as $r$ decreases. Since there are no visible crossings viewed from $\left(z_{0}, r_{0}\right)$, upon decreasing the first coordinate $r_{0}$ of this point to a suitable negative number $r_{1}$ to obtain the classical braid picture, we find that all crossings made by the $k$ th strand are now overcrossings. Therefore the $k$ th strand can be brought forward and separated from the rest of the braid. One sees by induction that the braid is trivial, and this proves the representation is faithful.

For the converse, assuming the Gassner representation of $B_{n+2}$ is faithful and $p$ is an arc of the required type in $\mathbb{C}-\{1, \ldots, n\}$, one wants to prove that each $w_{k}(\partial p)$ is an effective obstruction. Choose $k$, suppose $w_{k}(\partial p)=$ $0 .^{2}$ Of course, once there is a path $p$ with these properties, there is also, in $\mathbb{C}-\{1, \ldots, n+2\}$, a path $p$, of the required type which instead does not pass so far to the left as the integer 1 , never crosses the ray $\operatorname{Re}(z)=k+2, \operatorname{Im}(z)>0$, and does not terminate at $k+2$, with $w_{k+1}(\partial p)=0$. We will clearly be done once we show any such path can be isotoped, fixing the basepoint, not to meet the ray $\operatorname{Re}(z)=k+1, \operatorname{Im}(z)>0$ in $\mathbb{C}-\{1, \ldots, n+2\}$.

For such a path $p$, the proof will proceed by producing a braid with Gassner matrix 1 , such that the braid is trivial only if $p$ can be isotoped off the vertical ray at $k+1$. The first step is to choose a fairly simple braid, one of whose arcs projects to $p$. If $p$ connects $z_{0}$ to $j$, take the union of the vertical lines $z=1,2, \ldots,(j-1),(j+1), \ldots, n+2$ together with the graph of $p$, parametrized from $-\infty$ to 0 as $p$ goes from $z_{0}$ to $j$. To make the dangling ends agree with the lines $z=1,2, \ldots, n+2$ for large negative $r$, move the bottom end of the graph of $p$ in front of the first $j$, to position 2 , while moving the second to the $(j-1)$ st, trailing ends one unit to the right, as if the arcs were hanging from a ceiling. Call the resulting braid $a$. The arcs $p_{1}, \ldots, p_{n+2}$ now

\footnotetext{
${ }^{2}$ Note that by condition (b) this implies that $p$ does not terminate at $k$.
} 
have the property that $p_{2}$ is isotopic to $p$, so $w_{k+1}\left(\partial p_{2}\right)=w_{k+2}\left(\partial p_{2}\right)=0$.

For any $s$, let $\sigma_{s}$ denote the braid in which the $s$ th and $(s+1)$ st strands perform a half-twist in the counterclockwise direction as $r$ tends from $-\infty$ to 0 . The action of $\sigma_{s}$ on a general element of $W$ is to first interchange all occurrences of $\lambda_{s}$ and $\lambda_{s+1}$ in the coefficients, and to afterwards send the basis vector $e_{s}$ to $e_{s+1}, e_{s+1}$ to $T_{s+1} e_{s}+\left(1-T_{s}\right) e_{s+1}$. The formulas at the end of the previous paragraph show that the action of $\sigma_{k+1}$ on $w\left(\partial p_{2}\right)$ is trivial, except that all occurrences of $\lambda_{1}$ and $\lambda_{2}$ in the coefficients are interchanged. It follows that $a^{-1} \sigma_{k+1}^{2} a$ fixes $e_{2}$, and it clearly fixes $e_{1}$. It is easy to see now that for $b=a^{-1} \sigma_{k+1} a$ and any even number $s$ the commutator $\left[b^{s}, \sigma_{1}^{s}\right]$ fixes $e_{1}$ and $e_{2}$. On the other hand, for $k \geq 3$, writing

$$
b^{-s} e_{k}=c_{2} e_{2}+\cdots+c_{n+2} e_{n+2}, \quad \sigma_{1}^{s} e_{2}=d_{1} e_{1}+d_{2} e_{2},
$$

we have, still for $s$ even,

$$
\begin{aligned}
b^{s} \sigma_{1}^{s} b^{-s} \sigma_{1}^{-s} e_{k} & =b^{s} \sigma_{1}^{s} b^{-s} e_{k}=b^{s}\left(c_{2}\left(d_{1} e_{1}+\left(d_{2}-1\right) e_{2}\right)+b^{-s} e_{k}\right) \\
& =c_{2} d_{1} e_{1}+c_{2}\left(d_{2}-1\right) e_{2}+e_{k} .
\end{aligned}
$$

If we raise $b$ to any integer power, the action of the commutator will remain in the one-parameter group parametrized by $c_{2}$, and will be raised to a corresponding complex power. In particular, and invoking our assumption that the representation is faithful,

$$
1=\left[\left[b^{s}, \sigma_{1}^{s}\right],\left[b^{2 s}, \sigma_{1}^{s}\right]\right] \text { for } b=a^{-1} \sigma_{k+1} a .
$$

Now, since both $b$ and $\sigma_{1}$ are conjugate to half-twists, yet sufficiently high powers fail to generate a free group, they must commute by [11]. Now the argument is geometric. I claim that the commutator $\left[\sigma_{1}^{-1}, b\right]$ is trivial only if $p$ can be isotoped off the upward-pointing ray at $k+1$ in $\mathbb{C}-\{1, \ldots, n+2\}$. For, in the usual braid diagram, the first strand crosses behind the $(k+2)$ nd only in the second occurrence of $a$, of the four in the word $\sigma_{1}^{-1} a^{-1} \sigma_{k+1} a \sigma_{1} a^{-1} \sigma_{k+1}^{-1} a$. The only way the first strand can be brought to the front is if the segment within this occurrence of $a$ can be brought in front of the segment of the $(k+2)$ nd strand there. Viewing just the braid $a$ from above, as in the notation section, these segments appear as the arcs $p$ and $E_{k+1}$, and the problem of isotoping the two strands of the braid in an appropriate way so the one does not pass behind the other is equivalent to the question of whether, in $\mathbb{C}-\{1, \ldots, n+2\}, p$ can be isotoped away from the ray $\operatorname{Re}(z)=k+1, \operatorname{Im}(z)>0$. Since we have shown, using faithfulness, that the braid is trivial, it must then be possible to isotop the simple arc $p$ away from the vertical ray. This shows that the Fourier series $w_{k+1}(\partial p)$ is an effective obstruction for this special type of arc in $\mathbb{C}-\{1, \ldots, n+2\}$, and that therefore $w_{k}(\partial p)$ is an effective obstruction in $\mathbb{C}-\{1, \ldots, n\}$ as desired.

Remark 1. If one is not concerned with the precise range of faithfulness, it is not necessary to assume that $\gamma$ is the boundary of an arc $p$; one can take for $\gamma$ an arbitrary simple closed curve in $\mathbb{C}-\{1, \ldots, n\}$, as we have already stated in the paragraph preceding the Theorem, provided the basepoint $z_{0}$ has minimum real-coordinate value among the points of $\gamma$. For, we can break $\gamma$ at $z_{0}$, connecting one of the ends to $n+1$. Now, calling this path $p$, we have

$$
w_{k}(\partial p)=\left(1-e^{2 \pi i \lambda_{n+1}}\right) w_{k}(\gamma), \quad k=1, \ldots, n,
$$


where the $w_{k}$, calculated in $\mathbb{C}-\{1, \ldots, n\}$ or $\mathbb{C}-\{1, \ldots, n+1\}$, turn out to be the same. Now if $e^{2 \pi i \lambda_{n+1}}=1$, then all $e^{2 \pi i \lambda_{k}}=1$, as they are conjugate. In this case $w_{k}(\gamma)$ is not effective, and the representation is faithful for no value of $n$. If $e^{2 \pi i \lambda_{n+1}} \neq 1$, the condition whether $\gamma$ can be isotoped off the upwardpointing ray at $k$ in $\mathbb{C}-\{1, \ldots, n\}$, and the condition whether $w_{k}(\gamma)=0$, are equivalent to the analogous conditions for $\partial p$ in $\mathbb{C}-\{1, \ldots, n+1\}$.

Remark 2. Similarly, if one is not concerned with the precise range of faithfulness, one can always assume that the path or circle in question does not cross the downward-pointing $\operatorname{ray} \operatorname{Re}(z)=k, \operatorname{Im}(z)<0$.

\section{The Burau Representation}

The condition that $\gamma$ should be a simple loop, without self-intersections, plays an interesting role. In the Burau case, one can make a singular loop $\gamma$ with $w_{1}(\gamma)=0$ which cannot be isotoped off the upward-pointing ray at 1 , simply by drawing a figure eight (Figure 1).

If we break $\gamma$ in two places, as shown in Figure 2 on the next page, and in this way resolve the crossing, connecting the free ends to the integers 1 and $k+2$, we will have

$$
w_{k+3}(\partial(\gamma))=\left(-1+T^{-1}-1+T\right)+T^{k-1}\left(-1+T^{-1}-T^{-2}+T^{-1}\right),
$$

where $\partial(\gamma)$ is the loop tracing the boundary of a small regular neighborhood of the broken arc $\gamma$.

We also allow an isotopy of the broken curve which brings the integers from 2 to $k+1$ into the triangular region, as shown in Figure 2. Note that for $k=2$, $w_{k+3}(\partial(\gamma))=0$. This is because two extra poles are needed to compensate for the fact that $p \partial(\gamma)$ encloses two new integers, 1 and $k+2$. The existence of such a curve $\partial(\gamma)$ together with Remark 1 and part (ii) of the Theorem imply that the Burau representation of $B_{n}$ is unfaithful for

$$
n=(k+5)+1+2=10 \text {. }
$$

The situation can be visualized as follows (see also the figures in the announcement [9]): Thinking of the dots at $1,2,3,4,6$, and $7=k+5$ as projections of arcs perpendicular to the page, while an arc at $k+3=5$ lies a

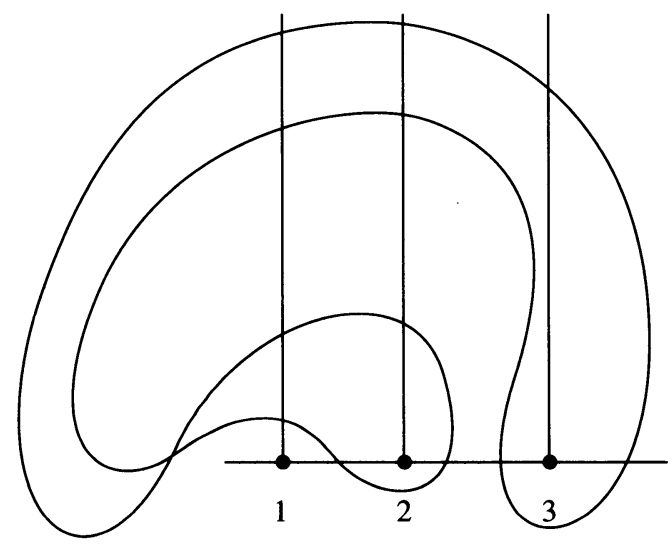

FiguRE 1 


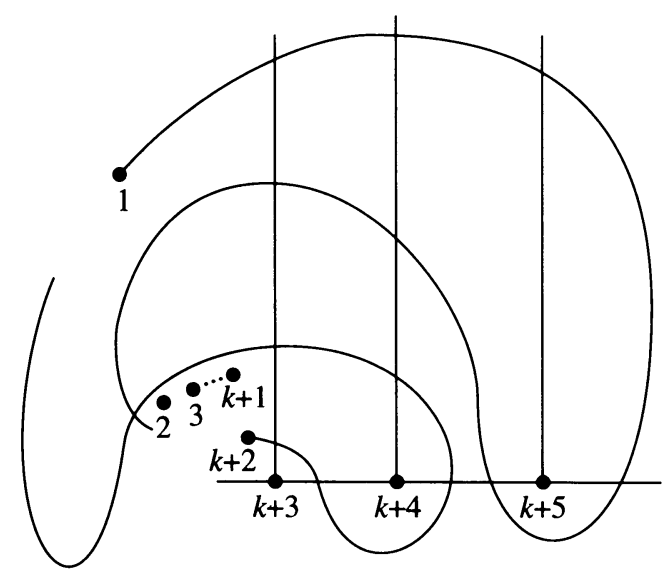

FigURE 2

bit below the page in the plane $\operatorname{Re}(z)=5$, and an additional arc loops about in the plane, one sees arcs in three perpendicular planes. Moving the arc at 5 within its plane from a level below the page to a level above represents a change of eight crossings. Although the entries of the Burau matrix concerning the more complicated strand do detect the other strand cutting through, they do so only for reasons of branch number, so those can be restored by moving any other strand through the same crossings in the other direction.

Part (ii) of the Theorem is meant to express what is, in this situation, the following construction: one makes a nontrivial braid by four applications of such a move, changing 16 crossings each time, beginning with a trivial braid on ten strands. Or, one changes a single crossing in a complicated way. One then uses the assumption of faithfulness to establish that the braid lies in the kernel.

Such mysterious logic is unsatisfying, and it leaves open the question, first raised by the referee, whether this braid does always lie in the kernel, rather than some unspecified braid. This open problem, and the problem created by the gap of two in the values of $n$ between parts (i) and (ii) of the Theorem, shall be brought to a satisfactory conclusion in the forthcoming paper of Long and Paton.

\section{ACKNOWLEDGMENTS}

I would like to take this opportunity to mention the sources of funding for this paper and [9], and two other manuscripts I have written in the last three years. In 1987, Professor W. Schelter helped afford me NSF grant number DMS 8704084, for a problem about group characters. In 1988, thanks to the hospitality of K. A. Brown at the University of Glasgow, we worked under SERC number GR/E/84204. In addition, K. A. Brown arranged for me to speak in a seminar, where I met $\mathrm{C}$. MacLachlin and S. Pride, and learned a great deal. This year we have been supported by an SERC grant arranged by the chairman of Queen Mary and Westfield College of the University of London.

I would like to thank Shaun Bullett for conveying to me the gist of some recent work on braids, which suggests avoiding the usual projections. Based on Witten's [10], Shaun suggested the possibility of obtaining knot invariants 
directly by integrating along the strands of a knot. This idea is followed in [9], and implicitly here, though we use the monodromy formulation of [8]. I would also like to thank Mark Paton for helpful conversations.

\section{REFERENCES}

1. J. W. Alexander, Topological invariants of knots, Trans. Amer. Math. Soc. 30 (1928), 275301.

2. W. Magnus, Collected papers (G. Baumslag and B. Chandler, eds.), Springer-Verlag, New York and Berlin, 1984.

3. D. D. Long, A note on the normal subgroups of mapping class groups, Math. Proc. Cambridge Philos. Soc. 99 (1986), 79-87.

4. A. A. Markov, Über die freié Äquivalenz geschlossener Zöpfe, Receuil Math. Moscou 1 (1935), 73-89.

5. J. Birman, Braids, links and mapping class groups, Ann. of Math. Stud., vol. 82, Princeton Univ. Press, Princeton, NJ, 1974.

6. J. W. Alexander, A lemma on systems of knotted curves, Proc. Nat. Acad. Sci. U.S.A. 9 (1923), 93-95.

7. D. D. Long, On the linear representation of braid groups, Trans. Amer. Math. Soc. 311 (1989), 532-559.

8. T. Kohno, Linear representations of braid groups and classical Yang Baxter equations, Contemp. Math., vol. 78, Amer. Math. Soc., Providence, RI, 1988, pp. 339-363.

9. J. Moody, The Burau representation of the braid group $B_{n}$ is unfaithful for large $n$, Bull. Amer. Math. Soc. (N.S.) 25 (1991), 379-384.

10. E. Witten, Quantum field theory and the Jones polynomial, Comm. Math. Phys. 121 (1989), 351-399.

11. J. McCarthy, $A$ Tits alternative for subgroups of surface mapping class groups, Trans. Amer. Math. Soc. 291 (1985), 583-612.

Mathematics Institute, University of Warwick, Coventry, ENGLANd CV4 7AL

E-mail address: MOODY@MATHS.WARWICK.AC.UK 